\title{
Difusión de prácticas de lucha contra la erosión en el olivar de la cuenca del Alto Genil Granadino
}

\author{
JAVIER CALATRAVA \\ Departamento de Economía de la Empresa, UNIVERSIDAD POLITÉCNICA DE \\ CARTAGENA, ESPAÑA. E-mail: j.calatrava@upct.es \\ JUAN AGUSTÍN FRANCO \\ Departamento de Economía, UNIVERSIDAD DE EXTREMADURA, ESPAÑA. E-mail: \\ franco@unex.es
}

\begin{abstract}
RESUMEN
Este trabajo analiza el proceso de difusión de prácticas de lucha contra la erosión en el olivar de la provincia de Granada utilizando información de un sondeo a agricultores. Las prácticas más extendidas son el no laboreo y el uso de restos triturados de poda, mientras que otras más tradicionales son minoritarias. Pese a su efectividad contra la erosión y a su inclusión en las exigencias de varios programas agroambientales, el uso de cubiertas vegetales es muy reducido. El motor de los procesos de difusión de las principales prácticas analizadas ha sido la interacción y la comunicación directa entre los agricultores.
\end{abstract}

Palabras clave: Erosión, conservación de suelos, olivar, modelos de difusión de tecnologías.

\section{Diffusion of Soil Erosion Control Practices in the Olive Orchards of the Alto Genil Basin (Granada, Spain)}

\begin{abstract}
This paper analyses the degree of adoption of different soil conservation practices in the olive groves of the Spanish province of Granada using data from a survey of olive farmers. No-tillage and mulch using the grinded pruning residues are the most widespread practices, while few farmers do other more traditional practices. Despite its effectiveness in reducing erosion and its inclusion in the requirements of several agri-environmental measures, the use of vegetated covers is quite reduced. The diffusion processes of the main conservation practices have been based on the interactions among farmers rather than in other external factors.
\end{abstract}

Keywords: Erosion, soil conservation, olive groves, diffusion of innovations models.

Clasificación JEL: Q12, Q24, C50

Artículo recibido en octubre de 2010 y aceptado en febrero de 2011

Artículo disponible en versión electrónica en la página www.revista-eea.net, ref. ə-29106 


\section{INTRODUCCIÓN}

Los procesos erosivos suponen una importante externalidad del proceso productivo de muchos sistemas agrarios así como una seria amenaza para su sustentabilidad, al reducir el potencial de producción agraria. Las prácticas de cultivo inadecuadas y la falta de mantenimiento de las estructuras de conservación del suelo acentúan la erosión y producen la degradación del suelo ${ }^{1}$. En el olivar la erosión del suelo constituye un serio problema con importantes impactos medioambientales, económicos y sociales (Milgroom et al., 2007). Una parte importante del olivar de Andalucía está situado en zonas con elevado nivel de riesgo de erosión. Aproximadamente el $36 \%$ de la superficie olivarera andaluza está situado en pendientes superiores al $15 \%$, principalmente en las provincias orientales (CAP, 2002). Pese a ello, el grado de realización de prácticas de conservación de suelos es aún reducido.

En la campaña 2006/2007 la superficie agrícola a nivel nacional en la que se realizaba una o más prácticas de agricultura de conservación era de 3.050 .000 hectáreas, lo que supone un $22,2 \%$ de las tierras de cultivo del país (Basch, 2009). En la mitad de dicha superficie se aplicaban técnicas de mínimo laboreo, mientras que en unas 700.000 hectáreas se utilizaba el no laboreo y en 850.000 hectáreas se mantenían cubiertas de vegetación en el suelo ${ }^{2}$. Según CalatravaRequena y González (2008), quienes analizan la adopción de varias innovaciones agrarias en el olivar andaluz, el 39\% de los olivicultores de la región realizaban no laboreo en 1999. Calatrava et al. (2007) estudian la adopción de prácticas de conservación de suelos en explotaciones olivareras de las provincias de Granada y Jaén, encontrando que en 2002 el $62 \%$ de los agricultores encuestados realizaban no laboreo, mientras que el $26 \%$ realizaban laboreo siguiendo las curvas de nivel. Otras prácticas como mantener

${ }^{1}$ Un factor determinante del nivel de erosión de un suelo son las prácticas de cultivo. Las prácticas de conservación de suelos más básicas (laboreo siguiendo curvas de nivel, coberturas del suelo, etc.) se centran en reducir el efecto de la pendiente del terreno. En pendientes elevadas no basta con estos métodos, siendo necesario modificar las laderas construyendo estructuras de conservación del suelo (terrazas, bancales, etc.)

${ }^{2}$ El laboreo de conservación y el no laboreo reducen de manera significativa la erosión con respecto a los sistemas tradicionales de laboreo (Milgroom et al., 2007). Su efectividad se incrementa cuando se combina con la construcción de terrazas o bancales y cuando se mantiene el mayor grado posible de cobertura del suelo utilizando vegetación o sus restos. Las cubiertas o las franjas de vegetación permiten reducir casi totalmente la pérdida de suelo en cultivos leñosos como el olivar (Francia-Martínez et al., 2006; Gómez et al., 2009), mientras que el uso de los restos triturados de la poda como acolchado permite controlar la erosión y reducir la pérdida de humedad y nutrientes del suelo (Rodríguez-Lizana et al., 2008). La evidencia empírica mostrada en los mencionados estudios sugiere que el uso de cubiertas o franjas de vegetación es la mejor opción para minimizar la erosión, siendo los porcentajes de reducción de la pérdida de suelo mayores que los alcanzados mediante el uso de los restos triturados de las labores de poda. 
vegetación en los bordes de las parcelas apenas eran realizadas por un 5\% de los agricultores.

Uno de los factores que ha podido favorecer el crecimiento de las prácticas de conservación de suelos en la agricultura española ha sido la creciente incorporación de criterios ambientales en la política agraria y de desarrollo rural comunitaria a través de los Códigos de Buenas Prácticas Agrarias, los programas agro-ambientales y, más recientemente, el sistema del pago único (Calatrava et al., 2011). En concreto, los agricultores que participaron en los Programas de Desarrollo Rural del período 2000-2006, vigente en el momento de realizarse esta encuesta, debían de cumplir con las Buenas Prácticas Agrarias establecidas a nivel nacional que, para cultivos leñosos como el olivar, contemplan la obligación de no labrar siguiendo la línea de máxima pendiente. En el caso concreto del programa agro-ambiental de lucha contra la erosión en suelos frágiles, los olivicultores participantes debían de mantener vegetación natural en las líndes de las parcelas, así como los muretes de piedra, setos o terrazas existentes, y mantener franjas de vegetación entre líneas (cobertura del $50 \%$ ) en pendientes de más del $8 \%$. En el actual período de programación 20072013, la Junta de Andalucía ha eliminado este programa integrándolo en otros como el de agricultura ecológica, el de producción integrada en olivar o el de agricultura de conservación en cultivos herbáceos.

Posteriormente, los Reglamentos CE No 1782/2003 y CE No 73/2009, que deroga al anterior, sobre el desacoplamiento de los pagos directos y la condicionalidad de dichas ayudas, han establecido una serie de prácticas agrarias relacionadas con el mantenimiento de las tierras en buenas condiciones agrarias y ambientales que se refieren, entre otras cuestiones, a la protección del suelo contra la erosión. A nivel nacional, estas medidas se establecen en el Real Decreto 2352/2004, de 23 de diciembre, y en el posterior Real Decreto 486/2009, de 3 de abril. Entre los requisitos a cumplir para recibir los pagos directos destacan tres condiciones exigibles para evitar la erosión del suelo: a) La construcción y mantenimiento en buen estado de las terrazas, ribazos y caballones; b) La cobertura mínima del suelo, que en olivar supone el mantenimiento de una cubierta vegetal en las calles transversales a la línea de máxima pendiente; c) La prohibición de tipo alguno de laboreo en pendientes superiores al 15\%, excepto para los cultivos en terrazas, si se realiza laboreo de conservación, si se mantiene una cobertura total del suelo, y en parcelas de menos de una hectárea.

Este trabajo analiza el grado de adopción y las características del proceso de difusión en el tiempo de diferentes prácticas de lucha contra la erosión en el olivar de la cuenca alta del Río Genil en la provincia de Granada, una de las principales zonas productoras del país pero también una de las más montañosas y sujetas a un mayor riesgo de erosión. A continuación se expone brevemente el 
marco conceptual del análisis de difusión y se comentan tanto la fuente de los datos utilizados como la metodología empleada en el análisis, para terminar con la exposición de los principales resultados y conclusiones de este trabajo.

\section{ANÁLISIS DE LA DIFUSIÓN AGREGADA DE INNOVACIONES AGRARIAS}

El término "difusión" hace referencia al nivel agregado de adopción de una tecnología o innovación en un momento específico de tiempo (Feder et al., 1985). El número de individuos o empresas que utilizan una determinada tecnología, tanto en términos relativos como absolutos, es una medida del grado de difusión o propagación de dicha tecnología en ese momento. La mayoría de los trabajos empíricos sobre la adopción agregada de tecnologías por empresas analizan el patrón temporal de la adopción para identificar las tendencias específicas en el ciclo de la difusión de la tecnología (Feder y Umali, 1993).

El trabajo que asentó las bases metodológicas del análisis de difusión de innovaciones fue el de Ryan y Gross (1943), quienes analizan el proceso de difusión del uso de la semilla de maíz híbrido entre los agricultores de Iowa, descubriendo que tal innovación podía modelizarse a través de una curva sigmoide de tipo logístico. Posteriormente, Griliches (1957 y 1960) desarrolla un modelo econométrico de difusión, basado en una función logística, para explicar las diferencias observadas a lo largo del tiempo en la adopción por los agricultores del maíz híbrido en varios estados norteamericanos. De sus resultados se concluía que a largo plazo las variables sociológicas tienden a cancelarse entre sí, prevaleciendo sólo las variables económicas como factores determinantes del patrón de cambio tecnológico.

Pese a la importancia dada a la rentabilidad de la tecnología en la mayoría de los estudios realizados desde los trabajos de Griliches, otros aspectos como las características de la tecnología y cómo ésta es percibida por los potenciales adoptantes, juegan también un importante papel en los procesos de difusión (Rogers, 1995). De hecho, el modelo de difusión de innovaciones basado en curvas logísticas se ajusta bien a procesos de difusión cuyo motor principal es el contacto entre los individuos del sistema en que se difunde y en los que la información circula de unos a otros y la innovación se adopta por contagio o imitación.

Otra explicación alternativa sobre la forma sigmoidal de la dinámica conjunta de adopción de innovaciones es la basada en diversas fuentes de heterogeneidad que suponen una dinámica gradual en el proceso de adopción (Stoneman, 1981). Así, entre las múltiples fuentes de heterogeneidad agraria cabe citar como principales: el tamaño de la explotación, la cualificación del capital humano, la capacidad de gestión, los rendimientos de los cultivos y los tipos de suelos (Abadi y Pannell, 1999). Otros autores señalan asimismo como 
posibles fuentes de heterogeneidad las preferencias por el riesgo (Leathers y Smale, 1991; Feder y Umali, 1993) y las percepciones de los agricultores (Marra et al., 2003).

Los modelos de difusión temporal describen la senda de adopción acumulada de una determinada tecnología o innovación a lo largo del tiempo, siendo también válidos para propósitos predictivos. Estos modelos analizan, por un lado, la penetración de una tecnología en su mercado potencial a lo largo del tiempo; y por el otro, el grado de saturación o nivel máximo de adopción. Tales análisis pueden hacerse desde dos ópticas complementarias: inter-empresas y global (Mahajan et al., 1990). La difusión inter-empresas estudia la evolución de la adopción a nivel agregado, es decir, el número de empresas/explotaciones que van tomando la decisión de adoptar. La difusión global se refiere a la intensidad de la adopción, es decir, el volumen de negocio bajo la adopción (por ejemplo, el número de hectáreas afectadas).

El modelo genérico de la tasa de difusión temporal puede expresarse como:

$$
\frac{d N(t)}{d t}=g(t)[M-N(t)]
$$

siendo: $\mathrm{N}(\mathrm{t})$ el número acumulado de adoptantes en el tiempo (curva de difusión); $\mathrm{g}(\mathrm{t})$ el coeficiente de difusión (determina el tipo de curva de difusión); y $\mathrm{M}$ el número máximo de potenciales adoptantes en el tiempo (grado de saturación).

Desde los ya comentados primeros trabajos que se basaban en curvas sigmoidales de adopción acumulada (Ryan y Gross, 1943; Rogers, 1958) se han propuesto diferentes formas funcionales según el tipo de innovación, distinguiéndose, según la procedencia de la información en que se basa la decisión de adoptar, entre modelos de influencia interna o logísticos (Griliches, 1957 y 1960; Mansfield, 1961), de influencia externa o exponenciales (Fourt y Woodlock, 1960) y mixtos o de influencia interna-externa (Bass, 1969).

Los modelos de influencia interna se basan en curvas sigmoidales (forma de S) que se ajustan bien a procesos de difusión basados en la imitación o efecto contagio, es decir, en el contacto entre los potenciales adoptantes del sistema en que se difunde la innovación. La información que se transmite entre los miembros del sistema social es de carácter interno, por lo que la difusión se produce por acumulación de información y experiencia, reduciéndose progresivamente la incertidumbre inicial a medida que los nuevos adoptantes "contagian" a los potenciales adoptantes (Mansfield, 1961). Estos modelos se adaptan bien al análisis de innovaciones en contextos sociales homogéneos donde el efecto de imitación es decisivo, puesto que implica que la decisión de no adoptar produce desventajas con respecto a los adoptantes (Feder y Umali, 1993). En contextos donde la adopción previa tiene una importancia mínima, donde la innovación es sencilla y no requiere etapas iniciales de aprendizaje, la 
difusión de innovaciones basada en información externa (medios de comunicación), sin que exista comunicación entre los miembros del sistema, se modeliza mejor a través de la función Exponencial, propuesta por Fourt y Woodlock (1960).

El modelo de Bass (1969) es una generalización de los modelos de influencia interna (logísticos) y externa (Exponencial). El modelo de Bass o de influencia mixta es adecuado para modelizar situaciones en las que el adoptante potencial se encuentra en contacto con otros agentes del sistema en que se difunde la innovación y además con los agentes externos que impulsaron inicialmente su adopción.

Aparte de las numerosas aplicaciones existentes en diversos ámbitos de las Ciencias Sociales, varios trabajos han utilizado el análisis de difusión para estudiar la adopción de tecnologías agrarias en España (Dios Palomares et al., 2003). Gómez Muñoz (1988) lo aplica al análisis de la difusión de nuevas variedades de trigo y girasol entre agricultores de la provincia de Córdoba. Carmona et al. (2005) lo aplican al análisis de la difusión de la agricultura ecológica en Murcia y Andalucía. Alcón et al. (2006) lo utilizan para estudiar el proceso de difusión del riego localizado en el Campo de Cartagena. Por su parte, Parra-López et al. (2007) analizan el proceso de difusión y la adopción de técnicas de agricultura ecológica en el olivar de cinco provincias andaluzas, concluyendo que dicho proceso de difusión se ha basado principalmente en la interacción y el "contagio" entre los agricultores, mientras que otros factores externos como los servicios públicos de extensión agraria no han resultado ser relevantes. Asimismo, Calatrava et al. (2007) encuentran evidencia empírica de un proceso de difusión sigmoidal de varias prácticas de lucha contra la erosión en explotaciones olivareras de diferentes municipios de las provincias de Granada y Jaén. El presente trabajo amplía y profundiza los resultados de dichos autores utilizando datos de un nuevo y más exhaustivo sondeo a explotaciones olivareras de la cuenca del Alto Genil, la principal zona olivarera de la provincia de Granada.

\section{METODOLOGÍA}

\subsection{Datos utilizados en el análisis}

Los datos utilizados en el análisis provienen de un sondeo a 215 explotaciones de olivar de los principales municipios olivareros de la provincia de Granada realizado en 2005. La zona de estudio está formada por 10 municipios del norte y del este de la provincia que pertenecen a la cuenca alta del Río Genil, la principal zona olivarera de Granada. El olivar es el principal cultivo de la provincia de Granada, ocupando 174.197 hectáreas $(41 \%$ de la tierra efectivamente cultivada, $28 \%$ del terreno agrícola y $14 \%$ de la superficie 
provincial). Los diez municipios suponen el $17 \%$ de la superficie de la provincia pero la mitad de su superficie olivarera (87.645 hectáreas) (CAP, 2006). El olivar supone el $75 \%$ de la tierra cultivada en el conjunto de los diez municipios y el $69,7 \%$ de la tierra agrícola tanto cultivada como no cultivada.

El tipo de explotación predominante en la zona es una explotación tradicional de secano extensivo y de pequeña superficie, si bien existe un pequeño número de explotaciones de elevado tamaño que suponen una parte significativa de la superficie olivarera. Asimismo, el número de explotaciones con sistemas de producción más intensivos está creciendo, como lo hace también la transformación a regadío.

Además de por ser representativa del olivar de la provincia de Granada, la zona fue seleccionada por estar afectada por un severo problema de erosión del suelo en las explotaciones agrarias. El grado de erosión en los suelos de la zona es superior al de los de la provincia, siendo reducido en el 5,4\% de la superficie, medio en el 25,3\%, alto en el 51,3\% y muy alto en el 18\% (CMA, 2002).

En el sondeo se preguntaba a los agricultores por: características de su explotación (superficie, pendientes, rendimientos, nivel de conservación del suelo, tipo de propiedad, etc.), percepción de la erosión por el agricultor, prácticas de conservación de suelos que realizan, uso de servicios de asesoramiento técnico, gestión de la explotación y características sociodemográficas (edad, nivel educativo, formación agraria, actitudes frente al riesgo, etc.). La muestra ha sido estratificada por municipio y superficie de la explotación utilizando datos del Censo Agrario (INE, 1999). El error estándar de muestreo es de $6,65 \%$ para la estimación de proporciones intermedias y de $2,91 \%$ para la de proporciones extremas.

\subsection{Modelos de difusión de innovaciones utilizados}

Los primeros trabajos sobre difusión de innovaciones (Ryan y Gross, 1943; Griliches, 1957 y 1960; Rogers, 1958) asumen que los procesos de difusión siguen patrones sigmoides. Pese a que la mayoría de estudios realizados desde entonces utilizan especificaciones Logísticas, Exponenciales o Normales, otras curvas sigmoides, tales como la Weibull o la Gompertz, son frecuentemente utilizadas. Menos utilizados son los modelos de Richards o Morgan-MercerFlodin (Ratkowsky, 1983) o las especificaciones polinómicas (Mahajan et al., 1988; Carrillo y González, 2002). Estas tienen la ventaja de su sencillez analítica, ya que pueden estimarse por métodos lineales, pero, al no existir una correspondencia entre los coeficientes del modelo y las características del proceso de difusión, apenas se utilizan.

Para el análisis de difusión de las prácticas de conservación de suelos identificadas en la zona de estudio se han estimado dos tipos de modelos de influencia interna, uno con una especificación simétrica de tipo Logístico o 
Verhulst-Pearl y otra asimétrica de tipo Gompertz, así como un modelo de influencia externa con una especificación de tipo Exponencial, y otro de tipo mixto con una especificación de Bass. En la Tabla 1 se muestran las respectivas especificaciones de los cuatro modelos estimados, que son los más importantes, representativos y utilizados dentro de la Teoría de la Difusión de innovaciones (Carmona Martínez y Gómez García, 2003).

El parámetro $b$ es el coeficiente de difusión, conocido también como coeficiente de imitación en las especificaciones sigmoides, que indica la velocidad del proceso de difusión. El parámetro $\mathrm{M}$ es el máximo porcentaje de adoptantes de la tecnología. El cociente $b / M$ puede interpretarse como el porcentaje de no-adoptantes "contagiados" en un diferencial de tiempo $d t$ por cada adoptante. Integrando la función de densidad se obtiene la función de distribución acumulada correspondiente. El parámetro $a$ es la constante de integración, donde mayores valores del mismo indican menores niveles iniciales del proceso. En los modelos sigmoides, tales como el Logístico y el Gompertz, la máxima tasa de adopción $(d N(t) / d t=0)$ ocurre en el punto de inflexión, es decir, cuando la innovación ha alcanzado la mediana (Banks, 1994). La función de probabilidad acumulada Logística es simétrica $\left(N\left(t^{*}\right)=50 \%\right)$, mientras que la función de distribución acumulada Gompertz es asimétrica y el punto de inflexión ocurre antes que en la curva logística $\left(N\left(t^{*}\right)=36,78 \%\right)$.

Tabla 1

Funciones de densidad y probabilidad acumulada de los modelos estimados

\begin{tabular}{|c|c|c|c|}
\hline Distribución & $\begin{array}{c}\text { Función de densidad } \\
n(t)=d N(t) / d t\end{array}$ & $\begin{array}{c}\text { Función de } \\
\text { distribución } \\
\text { acumulada } N(t)\end{array}$ & $\begin{array}{l}\text { Punto de inflexión } \\
\qquad d N(t) / d t=0\end{array}$ \\
\hline Logística & $b N(t)[M-N(t)]$ & $M /[1+e x p(a-b t)]$ & $\begin{array}{l}t^{*}=a / b \\
N\left(t^{\star}\right)=M / 2\end{array}$ \\
\hline Gompertz & $b N(t)[L n M-L n N(t)]$ & $M \exp [-\exp (a-b t)]$ & $\begin{array}{l}t^{\star}=(1 / b) \operatorname{Ln}\left[\operatorname{Ln}\left(M / N_{0}\right)\right] \\
N\left(t^{*}\right)=M / \exp \end{array}$ \\
\hline Exponencial & $b[M-N(t)]$ & $M[1-\exp (a-b t)]$ & No tiene \\
\hline Bass & $\begin{array}{c}p[M-N(t)]+q[N(t) / M][ \\
M-N(t)]\end{array}$ & $\begin{array}{l}{[M(1-\exp ((-p-q) t))] /} \\
{[1+(q / p) \exp ((-p-q) t)]}\end{array}$ & $\begin{array}{l}t^{*}=(-\operatorname{Ln}(p / q)) /(p+q) \\
N\left(t^{*}\right)=M(1 / 2-p / 2 q) .\end{array}$ \\
\hline
\end{tabular}

Fuente: Elaboración propia a partir de Banks (1994).

Por el contrario, la función de difusión Exponencial tiene forma cóncava, con asíntota superior y sin punto de inflexión (Tabla 1). La tasa de difusión $b$ 
del modelo Exponencial depende sólo del número de adoptantes potenciales presentes en el tiempo $t$, no existiendo relación entre los adoptantes previos y los potenciales. La interpretación de los parámetros $a$ y $b$ es similar al caso del modelo logístico. Finalmente, en el modelo Bass el parámetro $p$ se denomina coeficiente de innovación o de influencia externa y el parámetro $q$ coeficiente de imitación o de influencia interna. La velocidad del proceso de difusión será mayor cuando mayores sean los valores de los parámetros $\mathrm{p}$ y q, y el punto de inflexión o máximo nivel de adopción se alcanza cuando $t^{*}=[-\operatorname{Ln}(p / q)] /(p+q)$ y $\mathrm{N}\left(\mathrm{t}^{*}\right)=\mathrm{M}(1 / 2-\mathrm{p} / 2 \mathrm{q})$.

El análisis de difusión de las prácticas de conservación de suelos consideradas se ha realizado desde dos enfoques, según agricultores (difusión inter-empresas) y según superficie (difusión global). La difusión inter-empresas representa el porcentaje acumulado de agricultores que adoptan por primera vez una determinada práctica de conservación de suelos en cada periodo de tiempo, respecto al total de agricultores de la muestra. Por su parte, la difusión global mide los incrementos sucesivos porcentuales de superficie en la que se realiza cada práctica de conservación de suelos en cada periodo de tiempo. La variable explicativa en todos los modelos es el tiempo $t$ medido en años. Los modelos de difusión se han estimado por mínimos cuadrados no lineales (Greene, 2007), permitiendo que los coeficientes y el techo de adopción sean variables y se ajusten por sí mismos a la realidad (Banks, 1994).

\section{RESULTADOS}

\subsection{Características de las explotaciones de la muestra}

Si no se tienen en cuenta las explotaciones de la muestra que tienen más de 100 hectáreas, y que suponen un 5\% del total, la superficie media es de 5,63 hectáreas para las explotaciones de regadío y de 5,68 hectáreas para las de secano, siendo la diferencia no significativa $^{3}$. Si se incluyen dichas explotaciones la superficie media de las explotaciones sube a 37,49 hectáreas. El $86 \%$ de las explotaciones encuestadas son de secano, mientras que el $14 \%$ son de regadío.

La pendiente media es superior al $15 \%$ en el $42 \%$ de las explotaciones encuestadas, entre el 8 y el $15 \%$ en el $43 \%$ de las mismas, e inferior al $8 \%$ para el $15 \%$. Solamente el $3 \%$ de las parcelas están situadas en terrazas, mientras que el $9 \%$ se sitúa en zonas llanas y el $88 \%$ en laderas sin abancalar, lo que se corresponde con el $86 \%$ de las explotaciones con niveles medio-altos de pendiente.

\footnotetext{
${ }^{3}$ A priori podía esperarse que las explotaciones de regadío fuesen más pequeñas que las de secano, pero no es así porque se trata de explotaciones de secano que se han puesto en riego utilizando recursos subterráneos.
} 
Las explotaciones de la muestra son predominantemente explotaciones tradicionales extensivas de secano de carácter familiar. En concreto, la densidad de plantación media es de 104 árboles/ha, presentando el 90\% de la superficie una densidad de plantación inferior a 130 árboles/ha, lo que indica que se trata, en su mayoría, de explotaciones tradicionales, existiendo poco olivar intensivo ${ }^{4}$. La densidad media de plantación es superior en regadío (124 olivos/ha) que en secano (102 árboles/ha), aunque la mayoría del olivar de regadío de la muestra corresponde también a sistemas de producción extensivos. En el 77,68\% de las explotaciones encuestadas se utiliza mano de obra familiar aparte de la del titular, mientras que el 7,91\% utiliza mano de obra asalariada fija. Se trata, en este último caso, de las explotaciones de mayor tamaño, que son las que tienen dimensión suficiente para rentabilizar la contratación de trabajadores fijos. Mientras que la contratación de mano de obra fija está relacionada con un mayor tamaño de la explotación, el uso de mano de obra familiar se da, como era de esperar, en mayor medida en las explotaciones de menor superficie.

\subsection{Grado de difusión de las prácticas de lucha contra la erosión}

La Tabla 2 recoge los porcentajes de las prácticas de conservación de suelos empleadas en la zona de estudio. La práctica más difundida es la de no laboreo con aplicación de herbicidas, realizada por el $90 \%$ de los agricultores y en casi la totalidad de la superficie. El elevado porcentaje de agricultores que realizan no laboreo en la zona se explica principalmente por las altas pendientes y los elevados costes de labrar en pendientes elevadas. Hay que comentar que el no laboreo sin herbicidas utilizando desbrozadoras no es utilizado por ninguno de los encuestados, que lo perciben como una práctica poco efectiva y de mayor coste que la aplicación de herbicidas.

El 10\% de agricultores que continúa haciendo laboreo, lo realiza siguiendo las curvas de nivel, que es una de las "buenas prácticas agrarias" exigidas para participar en los diferentes programas de desarrollo rural establecidos en el Reglamento CE $\mathrm{N}^{\mathrm{o}} 1257 / 99$. Algunos de estos agricultores que todavía realizan laboreo, evitan también el uso de aperos que volteen el suelo como la vertedera o la grada de discos (Tabla 2), requisito que se establece de manera implícita en las "buenas prácticas agrarias" y de manera explícita en el programa agroambiental de lucha contra la erosión en suelos frágiles (Real Decreto 4/2001).

\footnotetext{
${ }^{4}$ La densidad de plantación es una medida del grado de intensificación de la explotación. La Junta de Andalucía (CAP, 2002) considera como olivar intensivo aquel que se ajusta a pautas de aumento de densidad de plantación, por encima de los 140 árboles/ha, y que se adecua a la recolección mecánica mediante vibrador de tronco.
} 
Tabla 2

Prácticas de lucha contra la erosión adoptadas en la zona de estudio

\begin{tabular}{|c|c|c|c|}
\hline Prácticas & $\begin{array}{l}\text { Porcentaje de } \\
\text { explotaciones }\end{array}$ & $\begin{array}{l}\text { Porcentaje de } \\
\text { superficie }\end{array}$ & $\begin{array}{c}\text { Año de } \\
\text { inicio }\end{array}$ \\
\hline Laboreo & 10,23 & 0,72 & \\
\hline Sólo laboreo según curvas de nivel & 3,26 & 0,15 & \\
\hline $\begin{array}{l}\text { Laboreo según curvas de nivel y sin } \\
\text { uso de aperos que volteen el suelo }\end{array}$ & 6,98 & 0,57 & \\
\hline No laboreo con herbicidas & 89,77 & 99,28 & 1979 \\
\hline Triturado y mantenimiento de restos de poda & 42,79 & 93,18 & 1985 \\
\hline Prácticas & $\begin{array}{l}\text { Porcentaje de } \\
\text { explotaciones }\end{array}$ & $\begin{array}{l}\text { Porcentaje de } \\
\text { superficie }\end{array}$ & $\begin{array}{c}\text { Año de } \\
\text { inicio }\end{array}$ \\
\hline $\begin{array}{l}\text { Mantenimiento de vegetación natural en los } \\
\text { bordes de las parcelas }\end{array}$ & 10,70 & 37,78 & 1963 \\
\hline $\begin{array}{l}\text { Mantenimiento de setos en los bordes de las } \\
\text { parcelas }\end{array}$ & 8,37 & 37,34 & 1963 \\
\hline Cubiertas/franjas de vegetación en las calles & 4,19 & 0,30 & 1998 \\
\hline
\end{tabular}

Nota: respuestas múltiples, porcentajes sobre el total de la muestra.

Una práctica complementaria al no laboreo, y que se encuentra muy difundida en la cuenca del Alto Genil (43\% de los encuestados), es el mantenimiento de restos de poda sobre el suelo, que comenzó a adoptarse a mediados de los 80 . Las prácticas más antiguas son las de mantenimiento de vegetación natural y setos en las lindes de las parcelas, que datan de los años 60, si bien en la actualidad son realizadas por un pequeño número de agricultores.

De los porcentajes de adopción obtenidos (Tabla 2) puede deducirse que el laboreo es practicado todavía por una minoría de explotaciones de pequeño tamaño. Asimismo, el triturado de restos de poda y el mantenimiento de vegetación natural y/o setos en las lindes de las parcelas lo realizan las explotaciones de mayor tamaño, hecho que parece estar relacionado directamente con la disponibilidad de mano de obra fija en la explotación.

\subsection{Análisis de los procesos de difusión}

Antes de entrar a comentar los modelos de difusión estimados, hay que indicar que en el caso de algunas prácticas minoritarias (vegetación natural o setos en los bordes de las parcelas, cubiertas vegetales) el nivel de difusión actual es bastante reducido, y los resultados de los modelos estimados deben de tomarse con la debida cautela, como se comentará más adelante. Asimismo, es necesario indicar que no se ha estimado modelo alguno para las prácticas de laboreo siguiendo curvas de nivel y sin uso de aperos que volteen el suelo. Se trata de prácticas realizadas por el escaso número de agricultores que no han adoptado no laboreo en la zona, y cuya difusión está limitada por lo extendido de dicha práctica. 
En las Figuras 1 a 5 pueden verse las diferentes curvas del proceso de difusión de las principales prácticas de lucha contra la erosión en la zona de estudio tanto en términos de su difusión inter-empresas (línea continua) como de su difusión global (línea discontinua). Se observa cómo el proceso de difusión es más rápido en términos de superficie que en términos de agricultores adoptantes para la mayoría de las prácticas analizadas, lo que quiere decir que las explotaciones de mayor tamaño han adoptado antes dichas prácticas. Es el caso del mantenimiento de vegetación natural y setos en las lindes de las parcelas, con un retraso de la difusión inter-empresas de 5 y 9 años respectivamente, y del uso de restos de poda triturados, con un retraso medio de 6 años.

Los diferentes modelos de difusión estimados para las prácticas de conservación de suelos analizadas en la zona de estudio se recogen en las Tablas 3 a 6, en las que aparecen los coeficientes de los parámetros estimados ( $a, b, M$ y los parámetros $p$ y $q$ del modelo Bass), el coeficiente de determinación $\mathrm{R}^{2}$ como medida de bondad del ajuste, los puntos de inflexión en los modelos de influencia interna $\left(\mathrm{t}^{*}\right)$, y los valores del punto de partida o instante inicial $\left(\mathrm{t}_{0}\right)$. Además de los coeficientes estimados, se comentan también los coeficientes de penetración de las prácticas de lucha contra la erosión analizadas que se muestran en la Tabla 7.

Figura 1

Difusión inter-empresas y global del no laboreo

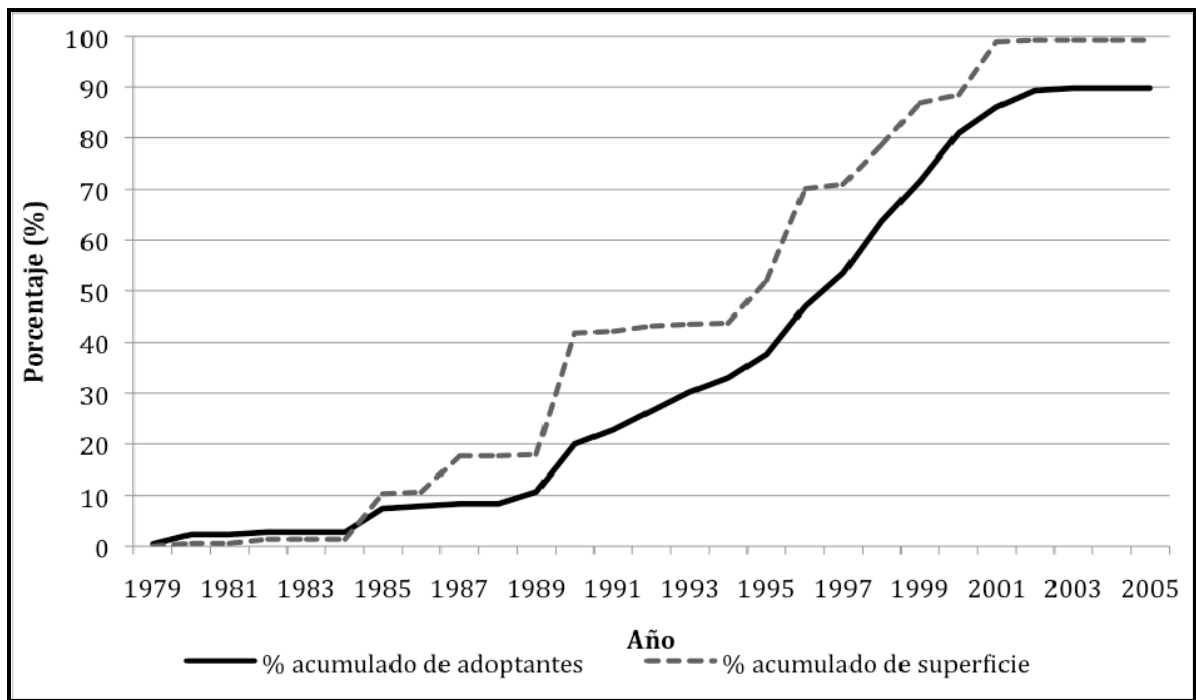


Figura 2

Difusión inter-empresas y global del uso de restos de poda triturados

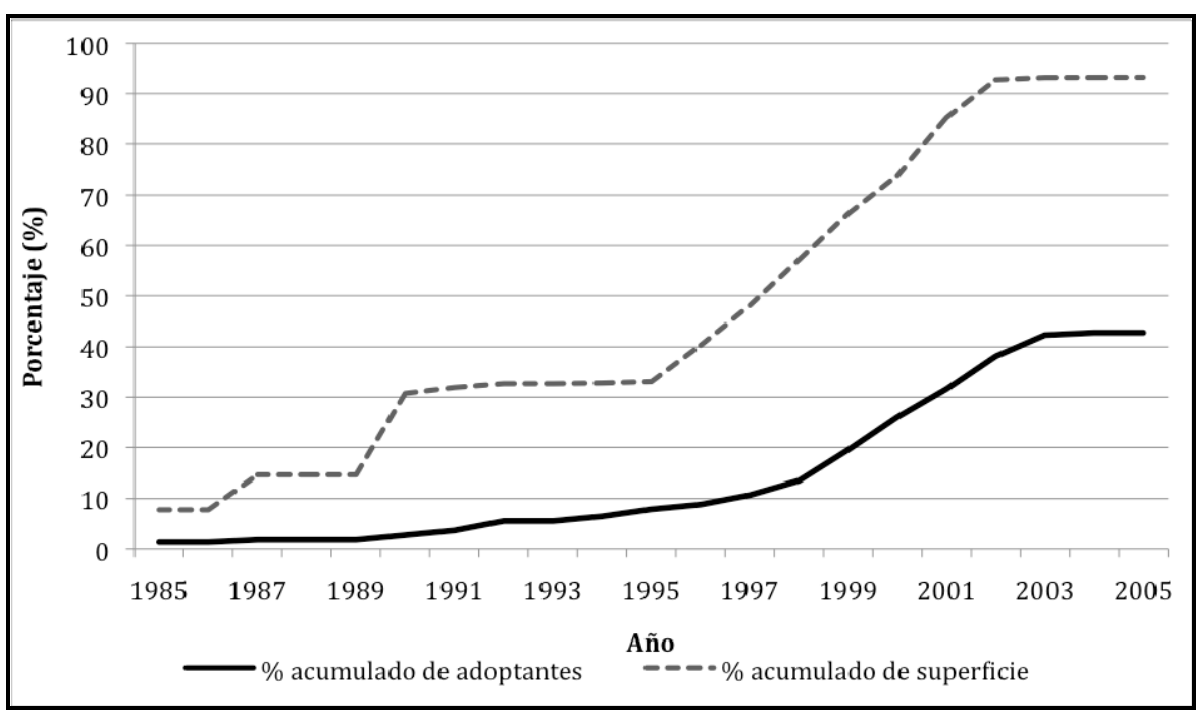

Figura 3

Difusión inter-empresas y global del mantenimiento de vegetación natural en las lindes

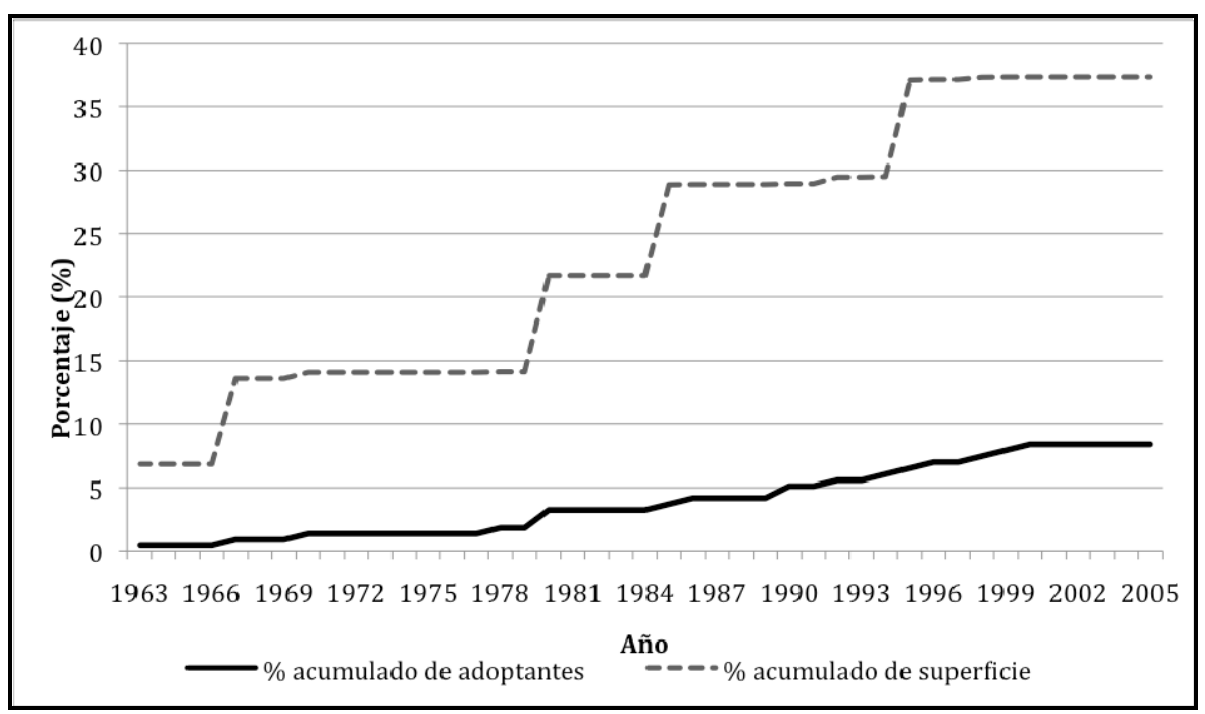


Figura 4

Difusión inter-empresas y global del establecimiento de setos en las lindes

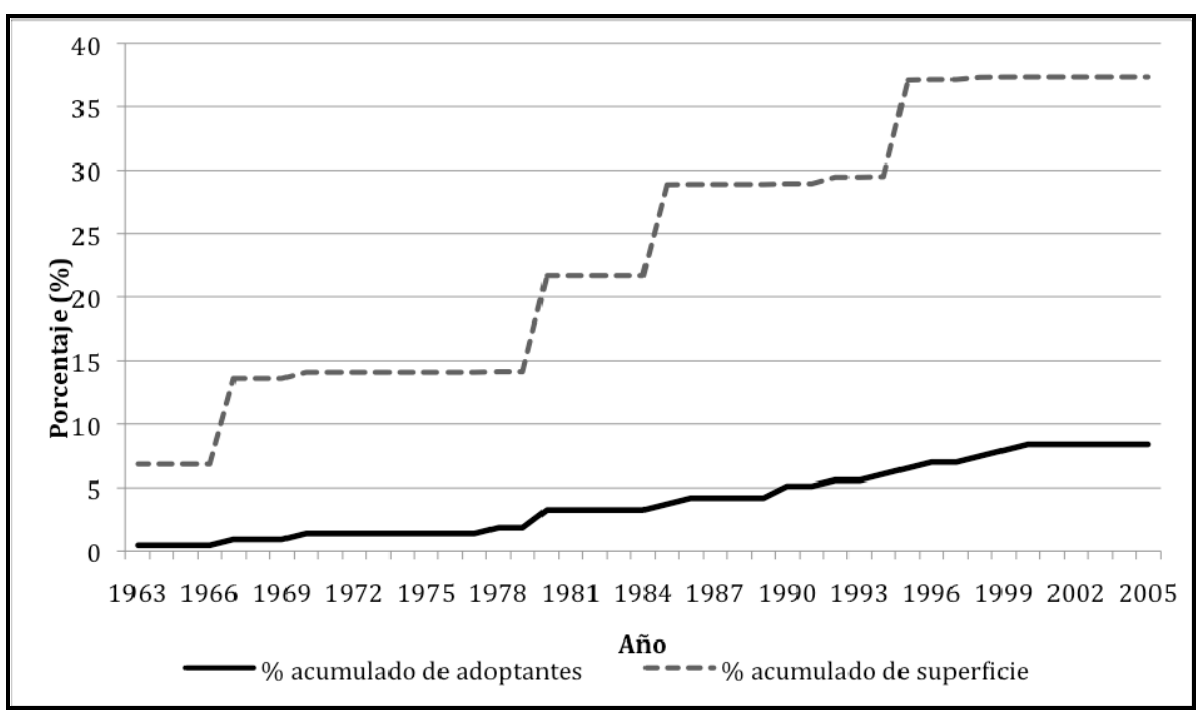

Figura 5

Difusión inter-empresas y global del mantenimiento de cubiertas vegetales

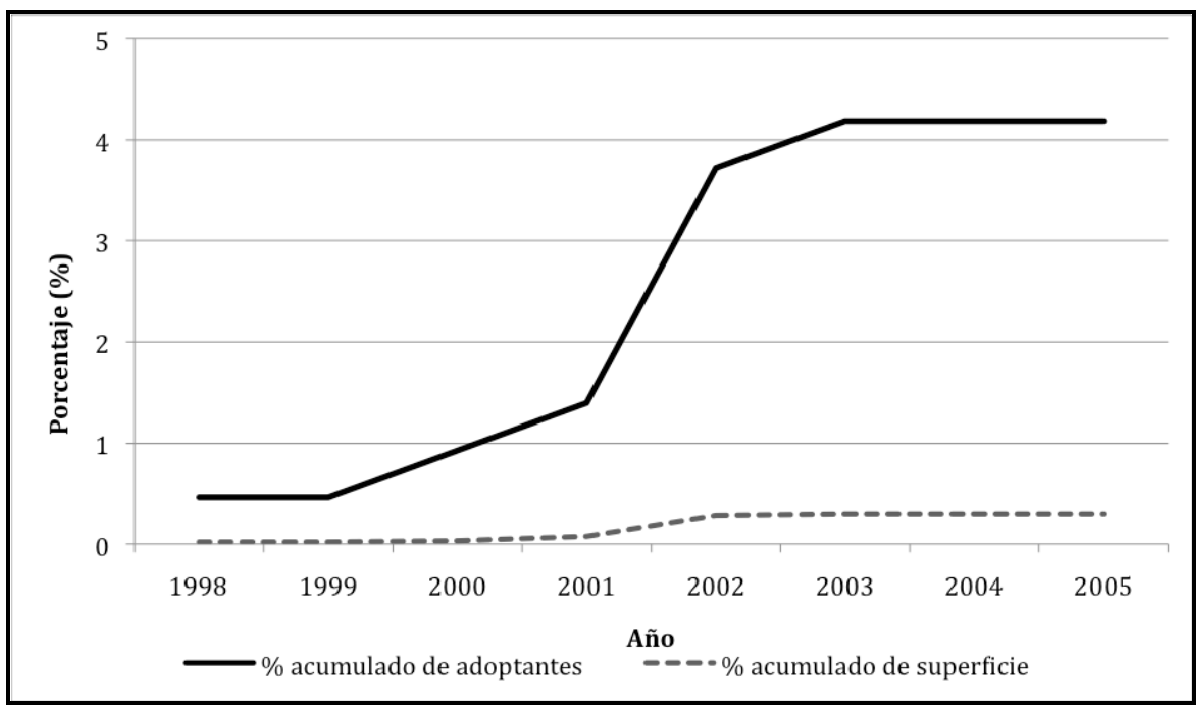

Para todas las prácticas analizadas, los modelos de influencia interna presentan mejor ajuste que los de influencia externa, ya que en casi todos el coeficiente de determinación supera el 0,95, tanto a nivel de agricultores como 
de superficie. La bondad de ajuste es ligeramente superior en los modelos Logísticos (Tabla 3) que en los Gompertz (Tabla 4), mientras que la bondad de ajuste de los modelos de tipo Exponencial (Tabla 5), si bien es alta, es bastante inferior a la de los dos modelos de influencia interna estimados. Los resultados de los modelos de Bass (Tabla 6) son similares a los modelos Logísticos. Los valores del coeficiente de innovación $p$ de los modelos Bass son muy reducidos, cero en algunos casos, en relación con el coeficiente de imitación $q$. De hecho, los valores del coeficiente de imitación q de los modelos Bass estimados (Tabla 6) son similares, si no idénticos, a los del coeficiente $b$ de los modelos Logísticos (Tabla 3). Puesto que son los modelos Logísticos los que presentan mejores ajustes, nos centraremos en ellos para profundizar en el estudio de las características del proceso de difusión de las prácticas identificadas en la zona de estudio.

Tabla 3

Modelos Logísticos de difusión de las prácticas de conservación de suelos

\begin{tabular}{|c|c|c|c|c|c|c|c|}
\hline \multirow[t]{2}{*}{ Práctica } & \multirow[t]{2}{*}{ Difusión } & \multicolumn{3}{|c|}{$\begin{array}{l}\text { Parámetros estimados* } \\
\text { (y error típico) }\end{array}$} & \multirow{2}{*}{$\begin{array}{c}\begin{array}{c}\text { Bondad } \\
\text { del } \\
\text { ajuste }\end{array} \\
R^{2} \\
\end{array}$} & \multicolumn{2}{|c|}{$\begin{array}{l}\text { Punto de inflexión } \\
\text { (y punto inicial) }\end{array}$} \\
\hline & & M & a & b & & $\begin{array}{c}t^{*} \\
\left(t_{0}\right)\end{array}$ & $\begin{array}{c}N\left(t^{*}\right) \\
{\left[N\left(t_{0}\right)\right]}\end{array}$ \\
\hline \multirow{4}{*}{ No laboreo } & \multirow{2}{*}{$\%$ Agricultores } & 100,000 & 5,214 & 0,291 & \multirow{2}{*}{0,992} & \multirow{2}{*}{$\begin{array}{c}1997 \\
(1979)\end{array}$} & \multirow{2}{*}{$\begin{array}{l}50,00 \\
(0,47)\end{array}$} \\
\hline & & $(3,834)$ & $(0,261)$ & $(0,019)$ & & & \\
\hline & \multirow{2}{*}{ \% Superficie } & 100,000 & 4,445 & 0,290 & \multirow{2}{*}{0,977} & \multirow{2}{*}{$\begin{array}{c}1994 \\
(1979)\end{array}$} & \multirow{2}{*}{$\begin{array}{l}50,00 \\
(0,02)\end{array}$} \\
\hline & & $(4,824)$ & $(0,396)$ & $(0,032)$ & & & \\
\hline \multirow{4}{*}{$\begin{array}{l}\text { Triturado } \\
\text { de restos } \\
\text { de poda }\end{array}$} & \multirow{2}{*}{$\%$ Agricultores } & 67,409 & 5,482 & 0,311 & \multirow{2}{*}{0,987} & \multirow{2}{*}{$\begin{array}{c}2003 \\
(1985)\end{array}$} & \multirow{2}{*}{$\begin{array}{l}33,70 \\
(1,40)\end{array}$} \\
\hline & & $(10,192)$ & $(0,308)$ & $(0,033)$ & & & \\
\hline & \multirow{2}{*}{ \% Superficie } & 100,000 & 2,942 & 0,246 & \multirow{2}{*}{0,945} & \multirow{2}{*}{$\begin{array}{c}1997 \\
(1985)\end{array}$} & \multirow{2}{*}{$\begin{array}{l}50,00 \\
(7,80)\end{array}$} \\
\hline & & $(12,260)$ & $(0,316)$ & $(0,045)$ & & & \\
\hline \multirow{4}{*}{$\begin{array}{l}\text { Vegetación } \\
\text { natural en } \\
\text { lindes }\end{array}$} & \multirow{2}{*}{$\%$ Agricultores } & 12,747 & 3,116 & 0,118 & \multirow{2}{*}{0,992} & \multirow{2}{*}{$\begin{array}{c}1989 \\
(1963)\end{array}$} & \multirow{2}{*}{$\begin{array}{c}6,37 \\
(0,47) \\
\end{array}$} \\
\hline & & $(0,435)$ & $(0,081)$ & $(0,005)$ & & & \\
\hline & \multirow{2}{*}{ \% Superficie } & 46,830 & 1,766 & 0,083 & \multirow{2}{*}{0,956} & \multirow{2}{*}{$\begin{array}{c}1984 \\
(1963)\end{array}$} & \multirow{2}{*}{$\begin{array}{l}23,42 \\
(6,82)\end{array}$} \\
\hline & & $(3,561)$ & $(0,087)$ & $(0,009)$ & & & \\
\hline \multirow{4}{*}{$\begin{array}{l}\text { Setos en } \\
\text { lindes }\end{array}$} & \multirow{2}{*}{$\%$ Agricultores } & 11,405 & 3,152 & 0,104 & 88 & 1993 & 5,70 \\
\hline & & $(0,706)$ & $(0,079)$ & $(0,006)$ & 0,500 & $(1963)$ & $(0,47)$ \\
\hline & \% Sunerficie & 46,532 & 1,758 & 0,082 & 0.953 & 1984 & 23,27 \\
\hline & & $(3,702)$ & $(0,089)$ & $(0,009)$ & & $(1963)$ & $(6,82)$ \\
\hline & $\%$ Aaricultores & 4,462 & 5,956 & 1,411 & 0.960 & 2002 & 2,23 \\
\hline Cubi & & $(0,457)$ & $(1,792)$ & $(0,463)$ & & $(1998)$ & $(0,47)$ \\
\hline vegetales & \% Sunerficie & 0,300 & 15,591 & 3,640 & 0.985 & 2002 & 0,15 \\
\hline & & $(0,015)$ & $(5,288)$ & $(1,305)$ & & (1998) & $(0,02)$ \\
\hline
\end{tabular}

Nota: * Parámetros significativos al 95\% de confianza. Modelo logístico: $\mathrm{N}(\mathrm{t})=\mathrm{M} /\left(1+\exp \left(\mathrm{a}-\mathrm{b}^{\star} \mathrm{t}\right)\right)$ 
Se deduce, por tanto, que son los modelos de influencia interna los que mejor explican el proceso de difusión de las prácticas analizadas entre los agricultores de la zona, tratándose de un proceso de difusión basado en la interacción directa entre ellos. La componente de innovación en el proceso de difusión de las prácticas analizadas es muy reducida frente a la componente de imitación. De hecho, casi un tercio de los agricultores no visita la oficina comarcal agraria y de los dos tercios restantes la inmensa mayoría acude a ésta a resolver trámites administrativos, principalmente relacionados con las ayudas de la Unión Europea. Es más, para la resolución de sus problemas en la explotación, el $80 \%$ prefiere acudir a otros agricultores y el $70 \%$ a la cooperativa, siendo bastante elevado el grado de satisfacción con las soluciones así alcanzadas. La adopción de las prácticas analizadas no es sencilla, ya que supone cambios importantes en el proceso productivo y presenta un cierto grado de incertidumbre en sus resultados, por lo que la componente de imitación del comportamiento exitoso de otros agricultores cobra mayor relevancia frente a la simple recepción de información externa sobre las ventajas de dichas prácticas.

Tabla 4

Modelos Gompertz de difusión de las prácticas de conservación de suelos

\begin{tabular}{|c|c|c|c|c|c|c|c|}
\hline \multirow[t]{2}{*}{ Práctica } & \multirow[t]{2}{*}{ Difusión } & \multicolumn{3}{|c|}{$\begin{array}{l}\text { Parámetros estimados* } \\
\text { (y error típico) }\end{array}$} & \multirow{2}{*}{$\begin{array}{c}\begin{array}{c}\text { Bondad } \\
\text { del } \\
\text { ajuste }\end{array} \\
\mathbf{R}^{2} \\
\end{array}$} & \multicolumn{2}{|c|}{$\begin{array}{l}\text { Punto de inflexión } \\
\text { (y punto inicial) }\end{array}$} \\
\hline & & M & a & b & & $\begin{array}{c}t^{*} \\
\left(t_{0}\right)\end{array}$ & $\begin{array}{c}N\left(t^{*}\right) \\
{\left[N\left(t_{0}\right)\right]}\end{array}$ \\
\hline \multirow{4}{*}{ No laboreo } & \multirow{2}{*}{$\%$ Agricultores } & 100,000 & 3,005 & 0,192 & \multirow{2}{*}{0,977} & \multirow{2}{*}{$\begin{array}{c}1988 \\
(1979)\end{array}$} & \multirow{2}{*}{$\begin{array}{l}36,79 \\
(0,47)\end{array}$} \\
\hline & & $(8,396)$ & $(0,387)$ & $(0,030)$ & & & \\
\hline & \multirow{2}{*}{ \% Superficie } & 100,000 & 2,529 & 0,196 & \multirow{2}{*}{0,968} & \multirow{2}{*}{$\begin{array}{c}1990 \\
(1979)\end{array}$} & \multirow{2}{*}{$\begin{array}{l}36,79 \\
(0,02)\end{array}$} \\
\hline & & $(7,154)$ & $(0,363)$ & $(0,033)$ & & & \\
\hline \multirow{4}{*}{$\begin{array}{l}\text { Triturado de } \\
\text { restos de } \\
\text { poda }\end{array}$} & \multirow{2}{*}{$\%$ Agricultores } & 100,000 & 2,399 & 0,131 & \multirow{2}{*}{0,978} & \multirow{2}{*}{$\begin{array}{c}1996 \\
(1985) \\
\end{array}$} & \multirow{2}{*}{$\begin{array}{l}36,79 \\
(1,40) \\
\end{array}$} \\
\hline & & $(38,779)$ & $(0,291)$ & $(0,036)$ & & & \\
\hline & \multirow{2}{*}{$\%$ Superficie } & 100,000 & 1,426 & 0,157 & \multirow{2}{*}{0,912} & \multirow{2}{*}{$\begin{array}{c}1991 \\
(1985)\end{array}$} & \multirow{2}{*}{$\begin{array}{l}36,79 \\
(7,80) \\
\end{array}$} \\
\hline & & $(18,661)$ & $(0,266)$ & $(0,048)$ & & & \\
\hline \multirow{4}{*}{$\begin{array}{l}\text { Vegetación } \\
\text { natural en } \\
\text { lindes }\end{array}$} & \multirow{2}{*}{$\%$ Agricultores } & 17,275 & 1,415 & 0,054 & \multirow{2}{*}{0,992} & \multirow{2}{*}{$\begin{array}{c}1987 \\
(1963)\end{array}$} & \multirow{2}{*}{$\begin{array}{c}6,35 \\
(0,47)\end{array}$} \\
\hline & & $(1,216)$ & $(0,035)$ & $(0,004)$ & & & \\
\hline & \multirow{2}{*}{$\%$ Superficie } & 60,344 & 0,816 & 0,041 & \multirow{2}{*}{0,953} & \multirow{2}{*}{$\begin{array}{c}1982 \\
(1963)\end{array}$} & \multirow{2}{*}{$\begin{array}{l}22,20 \\
(6,82)\end{array}$} \\
\hline & & $(9,620)$ & $(0,048)$ & $(0,008)$ & & & \\
\hline \multirow{4}{*}{$\begin{array}{l}\text { Setos en } \\
\text { lindes }\end{array}$} & \multirow{2}{*}{$\%$ Agricultores } & 20,124 & 1,421 & 0,039 & 0.987 & 1997 & 7,41 \\
\hline & & $(3,485)$ & $(0,027)$ & $(0,005)$ & U, & $(1963)$ & $(0,47)$ \\
\hline & \% Superficie & 60,284 & 0,813 & 0,040 & 0950 & 1982 & 22,18 \\
\hline & & $(10,118)$ & $(0,051)$ & $(0,008)$ & & $(1963)$ & $(6,82)$ \\
\hline & $\%$ Aqricultores & 4,804 & 3,086 & 0,805 & 0.939 & 1999 & 1,77 \\
\hline Cubie & & $(0,952)$ & $(1,399)$ & $(0,405)$ & & $(1998)$ & $(0,47)$ \\
\hline ve & $\% S$ & 0,298 & 13,633 & 3,330 & 0.984 & 1998 & 0,11 \\
\hline & & $(0,016)$ & $(7,641)$ & $(1,910)$ & & (1998) & $(0,02)$ \\
\hline
\end{tabular}

Nota: * Parámetros significativos al 95\% de confianza. Modelo Gompertz: $N(t)=M^{*} \exp \left(-\exp \left(a-b^{\star} t\right)\right)$. 
El proceso de difusión del no laboreo se inicia en 1979, si bien no despega hasta mediados de los años 80 del pasado siglo, alcanzándose el $10 \%$ de agricultores adoptantes en 1989 (Figura 1). La máxima tasa de crecimiento $t^{*}$ se da en 1997 (Tabla 3), alcanzándose en el momento del sondeo una difusión del $90 \%$ de los encuestados. En la Figura 1 puede verse cómo el proceso de difusión inter-empresas va retrasado con respecto a la difusión global, lo que indica que el no laboreo fue adoptado antes por las explotaciones de mayor tamaño. En cuanto a los coeficientes de penetración del no laboreo (Tabla 7), se observa que por cada $1 \%$ de los agricultores (y de las hectáreas) que ya ha adoptado se "contagia" cada año a un $0,29 \%$ de los que aún faltan por adoptar, es decir, anualmente cada agricultor adoptante persuade a 0,14 de los individuos no adoptantes y por cada 100 hectáreas se adopta la práctica en 0,36 hectáreas.

Tabla 5

Modelos Exponenciales de difusión de las prácticas de conservación de suelos

\begin{tabular}{|c|c|c|c|c|c|c|c|}
\hline \multirow[t]{2}{*}{ Práctica } & \multirow[t]{2}{*}{ Difusión } & \multicolumn{3}{|c|}{$\begin{array}{l}\text { Parámetros estimados* } \\
\text { (y error típico) }\end{array}$} & \multirow{2}{*}{$\begin{array}{c}\begin{array}{c}\text { Bondad } \\
\text { del } \\
\text { ajuste }\end{array} \\
R^{2}\end{array}$} & \multicolumn{2}{|c|}{ Punto inicial } \\
\hline & & M & $\mathbf{a}$ & b & & $t_{0}$ & $N\left(t_{0}\right)$ \\
\hline \multirow{4}{*}{ No laboreo } & \multirow{2}{*}{$\%$ Agricultores } & 100,000 & 4,838 & 0,053 & \multirow{2}{*}{0,791} & \multirow{2}{*}{1979} & \multirow{2}{*}{0,47} \\
\hline & & $(44,680)$ & $(0,299)$ & $(0,036)$ & & & \\
\hline & \multirow{2}{*}{$\%$ Superficie } & 100,000 & 4,886 & 0,072 & \multirow{2}{*}{0,840} & \multirow{2}{*}{1979} & \multirow{2}{*}{0,02} \\
\hline & & $(25,424)$ & $(0,151)$ & $(0,032)$ & & & \\
\hline \multirow{4}{*}{$\begin{array}{l}\text { Triturado de } \\
\text { restos de } \\
\text { poda }\end{array}$} & \multirow{2}{*}{$\%$ Agricultores } & 100,000 & 4,702 & 0,024 & \multirow{2}{*}{0,770} & \multirow{2}{*}{1985} & \multirow{2}{*}{1,40} \\
\hline & & $(191,808)$ & $(1,702)$ & $(0,054)$ & & & \\
\hline & \multirow{2}{*}{$\%$ Superficie } & 100,000 & 4,734 & 0,077 & \multirow{2}{*}{0,833} & \multirow{2}{*}{1985} & \multirow{2}{*}{7,80} \\
\hline & & $(34,097)$ & $(0,243)$ & $(0,048)$ & & & \\
\hline \multirow{4}{*}{$\begin{array}{c}\text { Vegetación } \\
\text { natural en } \\
\text { lindes }\end{array}$} & \multirow{2}{*}{$\%$ Agricultores } & 39,671 & 3,715 & 0,008 & \multirow{2}{*}{0,968} & \multirow{2}{*}{1963} & \multirow{2}{*}{0,47} \\
\hline & & $(22,795)$ & $(0,549)$ & $(0,005)$ & & & \\
\hline & \multirow{2}{*}{ \% Superficie } & 100,000 & 4,564 & 0,011 & \multirow{2}{*}{0,945} & \multirow{2}{*}{1963} & \multirow{2}{*}{6,82} \\
\hline & & $(49,796)$ & $(0,509)$ & $(0,007)$ & & & \\
\hline \multirow{4}{*}{$\begin{array}{l}\text { Setos en } \\
\text { lindes }\end{array}$} & \multirow{2}{*}{ \% Agricultores } & 30,611 & 3,453 & 0,008 & 0941 & & \\
\hline & & $(25,844)$ & $(0,810)$ & $(0,008)$ & 0,941 & 1963 & 0,47 \\
\hline & \% Sunerficie & 97,808 & 4,541 & 0,011 & 0.942 & 1963 & 6.82 \\
\hline & \% superticle & $(49,768)$ & $(0,520)$ & $(0,007)$ & 0,942 & 1963 & 0,82 \\
\hline & $\%$ Agricultores & 90,032 & 4,510 & 0,009 & 0.883 & 1998 & 0.47 \\
\hline Cuk & $\%$ Agricuitores & $(2125,80)$ & $(23,367)$ & $(0,210)$ & 0,883 & 1998 & 0,47 \\
\hline vegetales & $0 c_{1}$ & 8,762 & 2,181 & 0,007 & $92+2+3$ & 8 & ? \\
\hline & $\%$ supenicie & $(318,946)$ & $(36,266)$ & $(0,255)$ & 0,831 & 1998 & 0,02 \\
\hline
\end{tabular}

Nota: * Parámetros significativos al 95\% de confianza. Modelo exponencial: $N(t)=M-\exp (a-b \star t)$. 
En la Figura 2 puede verse cómo el proceso de difusión del uso de los restos triturados de la poda como acolchado se inicia en 1985, si bien no despega hasta la segunda mitad de la década de los noventa, situándose el punto de inflexión del proceso de difusión en 2003 , y alcanzando un $43 \%$ de los agricultores en 2005. Se observa también cómo el proceso de difusión inter-empresas va notablemente retrasado con respecto a la difusión global, lo que puede verse también en el hecho de que el punto de inflexión se produce en 1997 para la curva de difusión global Logística y en 2003 para la de difusión inter-empresas (Tabla 3). Esto indica que el triturado de los restos de poda fue adoptado antes por las explotaciones de mayor tamaño. También se observa cómo el retraso de la difusión inter-empresas se incrementa en los últimos años del período analizado, lo que se debe a la adopción de la práctica por explotaciones de gran tamaño durante el primer lustro del presente siglo. En cuanto a los coeficientes de penetración de la práctica del triturado de restos de poda, se observa que por cada $1 \%$ de los agricultores adoptantes son contagiados anualmente un $0,46 \%$ de los no adoptantes, es decir, por cada agricultor que ya ha adoptado son persuadidos anualmente 0,21 de los que todavía no lo habían hecho (Tabla 7). En términos de superficie, por cada $1 \%$ en que se realiza esta práctica es contagiado cada año un $0,25 \%$ de la superficie en la que aún no se ha adoptado.

Tabla 6

Modelos Bass de difusión de las prácticas de conservación de suelos

\begin{tabular}{|c|c|c|c|c|c|c|c|}
\hline \multirow{2}{*}{ Práctica } & \multirow{2}{*}{ Difusión } & \multicolumn{3}{|c|}{$\begin{array}{l}\text { Parámetros estimados* } \\
\text { (y error típico) }\end{array}$} & \multirow{2}{*}{$\begin{array}{c}\begin{array}{c}\text { Bondad } \\
\text { del ajuste }\end{array} \\
\mathbf{R}^{2}\end{array}$} & \multicolumn{2}{|c|}{$\begin{array}{l}\text { Punto de inflexión } \\
\text { (y punto inicial) }\end{array}$} \\
\hline & & M & $\mathbf{p}$ & q & & $\begin{array}{c}t^{*} \\
\left(t_{0}\right)\end{array}$ & $N\left(t^{*}\right)\left[N\left(t_{0}\right)\right]$ \\
\hline \multirow{4}{*}{ No laboreo } & \multirow{2}{*}{$\%$ Agricultores } & 100,00 & 0,002 & 0,287 & \multirow{2}{*}{0,992} & \multirow{2}{*}{$\begin{array}{l}1996 \\
(1979)\end{array}$} & \multirow{2}{*}{$\begin{array}{l}49,65 \\
(0,47)\end{array}$} \\
\hline & & $(4,173)$ & $(0,001)$ & $(0,022)$ & & & \\
\hline & \multirow{2}{*}{ \% Superficie } & 100,00 & 0,004 & 0,279 & \multirow{2}{*}{0,977} & \multirow{2}{*}{$\begin{array}{c}1994 \\
(1979)\end{array}$} & \multirow{2}{*}{$\begin{array}{l}49,28 \\
(0,02)\end{array}$} \\
\hline & & $(5,176)$ & $(0,001)$ & $(0,037)$ & & & \\
\hline \multirow{4}{*}{$\begin{array}{l}\text { Triturado de } \\
\text { restos de } \\
\text { poda }\end{array}$} & \multirow{2}{*}{$\%$ Agricultores } & 66,693 & 0,001 & 0,31 & \multirow{2}{*}{0,986} & \multirow{2}{*}{$\begin{array}{c}2003 \\
(1985)\end{array}$} & \multirow{2}{*}{$\begin{array}{l}33,24 \\
(1,40)\end{array}$} \\
\hline & & $(11,119)$ & $(0,0001)$ & $(0,04)$ & & & \\
\hline & \multirow{2}{*}{ \% Superficie } & 100,00 & 0,016 & 0,212 & \multirow{2}{*}{0,93} & \multirow{2}{*}{$\begin{array}{c}1996 \\
(1985) \\
\end{array}$} & \multirow{2}{*}{$\begin{array}{l}46,23 \\
(7,80)\end{array}$} \\
\hline & & $(17,576)$ & $(0,005)$ & $(0,073)$ & & & \\
\hline \multirow{4}{*}{$\begin{array}{l}\text { Vegetación } \\
\text { natural en } \\
\text { lindes }\end{array}$} & \multirow{2}{*}{ \% Agricultores } & 14,633 & 0,008 & 0,079 & \multirow{2}{*}{0,992} & \multirow{2}{*}{$\begin{array}{c}1989 \\
(1963)\end{array}$} & \multirow{2}{*}{$\begin{array}{c}6,58 \\
(0,47)\end{array}$} \\
\hline & & $(1,056)$ & $(0,001)$ & $(0,005)$ & & & \\
\hline & \multirow{2}{*}{ \% Superficie } & 46,42 & 0,007 & 0,141 & \multirow{2}{*}{0,924} & \multirow{2}{*}{$\begin{array}{c}1983 \\
(1963)\end{array}$} & \multirow{2}{*}{$\begin{array}{l}22,06 \\
(6,82)\end{array}$} \\
\hline & & $(4,238)$ & $(0,003)$ & $(0,04)$ & & & \\
\hline \multirow{4}{*}{$\begin{array}{l}\text { Setos en } \\
\text { lindes }\end{array}$} & \multirow{2}{*}{$\%$ Agricultores } & 14,895 & 0,006 & 0,064 & \multirow{2}{*}{0,986} & \multirow{2}{*}{$\begin{array}{c}1996 \\
(1963)\end{array}$} & 6,75 \\
\hline & & $(1,091)$ & $(0,001)$ & $(0,012)$ & & & $(0,47)$ \\
\hline & & 46,27 & 0,012 & 0,093 & & $I 0$ & \\
\hline & $\%$ sup & $(5,427)$ & $(0,003)$ & $(0,033)$ & 0,9 & (1963) & (6 82) \\
\hline
\end{tabular}


Tabla 6 (continuación)

Modelos Bass de difusión de las prácticas de conservación de suelos

\begin{tabular}{|c|c|c|c|c|c|c|c|}
\hline \multirow{2}{*}{ Práctica } & \multirow{2}{*}{ Difusión } & \multicolumn{3}{|c|}{$\begin{array}{l}\text { Parámetros estimados* } \\
\text { (y error típico) }\end{array}$} & \multirow{2}{*}{$\begin{array}{c}\begin{array}{c}\text { Bondad } \\
\text { del ajuste }\end{array} \\
\mathbf{R}^{2} \\
\end{array}$} & \multicolumn{2}{|c|}{$\begin{array}{l}\text { Punto de inflexión } \\
\text { (y punto inicial) }\end{array}$} \\
\hline & & M & $\mathbf{p}$ & q & & $\begin{array}{c}t^{*} \\
\left(t_{0}\right)\end{array}$ & $N\left(\mathrm{t}^{\star}\right)\left[\mathrm{N}\left(\mathrm{t}_{0}\right)\right]$ \\
\hline \multirow{4}{*}{$\begin{array}{l}\text { Cubiertas } \\
\text { vegetales }\end{array}$} & \multirow{2}{*}{$\%$ Agricultores } & 4,443 & 0,003 & 1,449 & \multirow{2}{*}{0,959} & \multirow{2}{*}{$\begin{array}{c}2002 \\
(1998)\end{array}$} & \multirow{2}{*}{$\begin{array}{c}2,22 \\
(0,47)\end{array}$} \\
\hline & & $(0,453)$ & $(0,005)$ & $(0,507)$ & & & \\
\hline & \multirow{2}{*}{ \% Superficie } & 0,302 & $2,3310^{-6}$ & 3,286 & \multirow{2}{*}{0,985} & \multirow{2}{*}{$\begin{array}{c}2002 \\
(1998)\end{array}$} & \multirow{2}{*}{$\begin{array}{c}0,15 \\
(0,02)\end{array}$} \\
\hline & & $(0,015)$ & $(0,000)$ & $(1,004)$ & & & \\
\hline
\end{tabular}

Nota: * Parámetros significativos al 95\% de confianza.

Modelo Bass: $N(t)=\left[M^{*}\left(1-\exp \left((-p-q)^{\star} t\right)\right)\right] /\left[1+(q / p)^{*} \exp \left((-p-q)^{\star} t\right)\right]$

A continuación comentaremos de manera conjunta los resultados obtenidos del análisis de los procesos de difusión de las prácticas de mantenimiento de vegetación natural y setos en las lindes de las parcelas. El motivo es que son prácticas similares y que muchas de las explotaciones que realizan ambas prácticas son las mismas. Se trata de prácticas tradicionales pero minoritarias que algunos olivicultores afirman realizar desde la década de los sesenta del pasado siglo. Su grado de difusión ha crecido muy lentamente en las últimas décadas, suponiendo apenas el $10 \%$ de los agricultores. Si bien todos los modelos de difusión estimados presentan una elevada bondad del ajuste, siendo menores las diferencias entre los modelos de influencia interna y externa que en el caso de las otras prácticas analizadas, sus resultados deben de analizarse con la debida cautela por tratarse de procesos de difusión de alcance reducido y muy dilatados en el tiempo. El único resultado claro se refiere al elevado grado de concentración de estas prácticas en las explotaciones de mayor tamaño. Si tomamos los resultados de los modelos Logístico estimados para estas dos prácticas (Tabla 3), puede verse cómo en los respectivos puntos de inflexión aproximadamente el $6 \%$ de los agricultores realizaban estas prácticas, si bien dichos agricultores suponían el $23 \%$ de la superficie. En el momento de la encuesta, estas prácticas se realizaban en aproximadamente el $37 \%$ de la superficie (Tabla 2). En cuanto a los coeficientes de imitación y penetración, éstos son similares para ambas prácticas (Tabla 7). Se observa que por cada 1\% de los agricultores adoptantes, hay cada año un $0,90 \%$ de imitadores, es decir, por cada olivicultor que ya ha adoptado estas prácticas algo más de 0,40 agricultores son "contagiados" anualmente. Por cada $1 \%$ de la superficie en la que se han adoptado estás prácticas cada año se adoptan en el $0,18 \%$ de la superficie en que todavía no se realizaba. 
Tabla 7

Coeficientes de penetración (b/M) para las prácticas analizadas (modelo Logístico)

\begin{tabular}{|c|c|c|c|}
\hline \multicolumn{4}{|c|}{ NO LABOREO } \\
\hline \multicolumn{2}{|c|}{ Agricultores } & \multicolumn{2}{|c|}{ Superficie } \\
\hline por cada $1 \%$ & $0,29 \%$ & por cada $1 \%$ & $0,29 \%$ \\
\hline por cada 1 individuo & 0,14 & por cada 1 hectárea & 0,0036 \\
\hline \multicolumn{4}{|c|}{ TRITURADO DE RESTOS DE PODA } \\
\hline \multicolumn{2}{|c|}{ Agricultores } & \multicolumn{2}{|c|}{$\underline{\text { Superficie }}$} \\
\hline por cada $1 \%$ & $0,46 \%$ & por cada $1 \%$ & $0,25 \%$ \\
\hline por cada 1 individuo & 0,21 & por cada 1 hectárea & 0,0031 \\
\hline \multicolumn{4}{|c|}{ VEGETACIÓN NATURAL EN LINDES } \\
\hline \multicolumn{2}{|c|}{ Agricultores } & \multicolumn{2}{|c|}{ Superficie } \\
\hline por cada $1 \%$ & $0,93 \%$ & por cada $1 \%$ & $0,18 \%$ \\
\hline por cada 1 individuo & 0,43 & por cada 1 hectárea & 0,0022 \\
\hline \multicolumn{4}{|c|}{ SETOS EN LINDES } \\
\hline \multicolumn{2}{|c|}{ Agricultores } & \multicolumn{2}{|c|}{ Superficie } \\
\hline por cada $1 \%$ & $0,91 \%$ & por cada $1 \%$ & $0,18 \%$ \\
\hline por cada 1 individuo & 0,42 & por cada 1 hectárea & 0,0022 \\
\hline \multicolumn{4}{|c|}{ CUBIERTAS VEGETALES * } \\
\hline \multicolumn{2}{|c|}{ Agricultores } & \multicolumn{2}{|c|}{ Superficie } \\
\hline por cada $1 \%$ & $31,61 \%$ & por cada $1 \%$ & $12,69 \%$ \\
\hline por cada 1 individuo & 14,70 & por cada 1 hectárea & 15,04 \\
\hline
\end{tabular}

Nota (*): Los coeficientes del modelo de cubiertas vegetales son muy altos porque se trata de una práctica reciente y con pocos adoptantes.

Finalmente, la práctica de establecer cubiertas o franjas de vegetación es la menos adoptada de todas las identificadas en la zona. Se trata de la práctica más reciente, siendo 1998 el año de inicio de su difusión y 2002 el año en que su adopción fue mayor (Figura 5 y Tabla 3). Pese a ser una práctica muy efectiva para reducir la pérdida de suelo, los agricultores piensan que supone una importante competencia por agua y nutrientes con el cultivo y, en muchos casos, optan por la más popular opción del uso de restos triturados de las labores de poda. Puesto que el número de adoptantes es reducido y que se trata de una práctica relativamente reciente, los resultados de los modelos estimados para esta práctica deben de interpretarse teniendo esto en cuenta y tomarse con la debida cautela. Es de destacar que las explotaciones que la han adoptado son de pequeño tamaño y que, debido al reducido número de adoptantes, los coeficientes de imitación y penetración son elevados (Tabla 7).

Entre los factores que pueden explicar la popularidad del uso de los restos de poda como sistema de cobertura del suelo frente al uso de las cubiertas o franjas 
de vegetación, destaca la diferente percepción que los agricultores tienen de ambas prácticas. Aunque el uso de cubiertas vegetales es una práctica muy efectiva para reducir la escorrentía y la pérdida de suelo, los agricultores consideran que supone una importante competencia por el agua y los nutrientes con el cultivo, problema que no existe con el uso de restos vegetales triturados. Otro factor de relevancia es el coste de ambas prácticas. El uso de restos vegetales triturados procedentes de la poda es barato, entre otros motivos, porque los agricultores ya poseen el material y se ahorran también el coste de eliminar dichos restos. Además, y según cálculos de la Consejería de Agricultura y Agua de la Región de Murcia, su coste en pendientes superiores al $6 \%$ es inferior al de implantar y mantener coberturas vegetales en el suelo (CARM, 2007).

\section{CONCLUSIONES}

En este trabajo se ha analizado el grado de difusión de las prácticas de lucha contra la erosión utilizadas por los agricultores de las principales zonas olivareras de la provincia de Granada. De todas las prácticas identificadas en la zona, la más difundida es la de no laboreo con aplicación de herbicidas, que es utilizada por el $90 \%$ de los agricultores. Este elevado porcentaje se explica principalmente por las altas pendientes de la zona. El proceso de difusión del no laboreo en la zona se inició a principios de los años ochenta del pasado siglo, siendo especialmente intenso a partir de la segunda mitad de los noventa. Su difusión fue inicialmente más rápida entre explotaciones de menor tamaño, siendo las grandes explotaciones más tardías en adoptar esta técnica. Al tratarse actualmente de una práctica muy extendida, sus coeficientes de penetración son reducidos.

Una práctica complementaria al no laboreo muy difundida en la zona de estudio es el mantenimiento de restos triturados de poda sobre el suelo, que comenzó a adoptarse a mediados de los 80 , si bien no despega hasta la segunda mitad de la década de los noventa. En el momento de realizarse la encuesta era realizada por un $43 \%$ de los agricultores. La existencia de una adopción más temprana de esta práctica por parte de las explotaciones de mayor superficie y, por tanto, de un efecto de escala en el momento de la adopción, puede explicarse por los elevados costes de adquisición de la maquinaria para el triturado de los restos de poda. Los coeficientes de penetración de esta práctica en términos de agricultores adoptantes son superiores a los de la práctica de no laboreo, aunque similares a éstos en términos de superficie bajo esta práctica.

Las prácticas de mantenimiento de vegetación natural y setos en las lindes de las parcelas son bastante minoritarias. Su proceso de difusión ha sido lento y dilatado en el tiempo, suponiendo apenas el 10\% de los agricultores. Es destacable el elevado grado de concentración de estas prácticas en las 
explotaciones de mayor tamaño de la muestra, al igual que ocurre con la práctica de mantenimiento de los restos triturados de poda, estando este hecho relacionado directamente con el empleo de mano de obra fija en la explotación.

Finalmente, la práctica de establecer cubiertas o franjas de vegetación es la menos adoptada de todas las identificadas. Pese a ser muy efectiva para reducir la escorrentía y la pérdida de suelo los agricultores la perciben como una práctica que implica una importante competencia por agua y nutrientes con el cultivo. Se trata de una práctica relativamente reciente, realizada por un número reducido de agricultores de pequeñas o muy pequeñas explotaciones. Es de destacar que, debido al reducido número de adoptantes, los coeficientes de imitación y penetración son bastante elevados.

Pese a que las políticas que incluían la lucha contra la erosión entre sus objetivos en el momento de la encuesta no contemplaban las prácticas del no laboreo o del triturado de restos de poda entre los requerimientos técnicos exigidos a los agricultores, estas prácticas se han extendido bastante más que otras durante los últimos 15 años. Asimismo, y aunque el establecimiento de cubiertas o franjas de vegetación es una práctica incluida en los requerimientos técnicos de los programas agroambientales de conservación de suelos, su uso es mucho menor que el de la más popular práctica de proteger el suelo con los restos triturados de las labores de poda, práctica esta última que es percibida por los agricultores como más ventajosa y que tiene además un menor coste.

De los modelos de difusión agregada de prácticas de conservación del suelo estimados se concluye que el motor de los procesos de difusión de las principales prácticas de conservación de suelos en la zona de estudio ha sido la interacción y la comunicación directa entre los agricultores, siendo menor la influencia de agentes externos, tales como las políticas agrarias o los servicios de extensión agraria. Este resultado coincide con el encontrado por Parra-López et al. (2007) para el proceso de difusión de la agricultura ecológica en el olivar andaluz.

Que los agricultores hayan optado por algunas prácticas diferentes a las contempladas en las políticas, porque las perciben como más ventajosas basándose en las experiencias de otros agricultores, sugiere que el diseño de éstas no debe basarse solamente en criterios agronómicos y ambientales, sino también en un análisis previo del potencial de adopción a partir de las percepciones de los agricultores hacia las diferentes prácticas. Pese a esto, es indudable que las políticas existentes juegan un papel importante, al enviar a los agricultores el mensaje de que la conservación del medio ambiente en general, y de los suelos en particular, es un objetivo importante para la sociedad y susceptible de ser financiado. Además, las políticas pueden incentivar una adopción inicial por parte de algunos agricultores que puedan conocer y 
experimentar con las prácticas y difundir posteriormente su uso entre el resto de agricultores.

La efectividad de las políticas de subvenciones a las prácticas agrarias compatibles con el medio ambiente podría multiplicarse mediante la puesta en marcha de programas de formación y concienciación de los agricultores sobre las diferentes prácticas que pueden utilizarse para la conservación de suelos, así como sobre los beneficios que éstas y otras prácticas tienen para ellos y para la sociedad. Según nuestros resultados, los agricultores acuden principalmente a otros agricultores y a los técnicos de sus cooperativas en busca de asesoramiento técnico, un resultado encontrado también por Calatrava et al. (2011) en la Región de Murcia, por lo que éstos deberían jugar un papel activo en cualquier estrategia de formación e información a realizar.

\section{Agradecimientos}

Los autores agradecen sus comentarios y sugerencias a dos revisores anónimos. Javier Calatrava Leyva agradece al Ministerio de Educación y Ciencia su financiación a través del Proyecto AGL2006-12293-C02-02/AGR. Juan Agustín Franco agradece al Instituto Nacional de Investigación y Tecnología Agraria y Alimentaria (INIA) su financiación a través de una beca pre-doctoral.

\section{REFERENCIAS BIBLIOGRÁFICAS}

ABADI A.K. Y PANNELL D.J. (1999): "A conceptual framework of adoption of an agricultural innovation". Agricultural Economics, 21: pp 145-154.

ALCÓN, F.; DE MIGUEL, M.D. Y FERNÁNDEZ-ZAMUDIO, M.A. (2006): "Modelización de la difusión de la tecnología de riego localizado en el Campo de Cartagena". Revista Española de Estudios Agrosociales y Pesqueros, 210: pp 227-245.

BANKS R. (1994): Growth and diffusion phenomena: Mathematical frameworks and applications, New York: Springer-Verlag.

BASCH, G. (2009): "No-tillage Worldwide". General Assembly of the European Conservation Agriculture Federation, Helsinky. Disponible en: http://www.ecaf.org/. [Último acceso: febrero de 2011]

BASS, F.M. (1969): "A new product growth model for consumer durables". Management Science, 15: pp 215-227. 
CALATRAVA-REQUENA, J. Y GONZÁLEZ, M.C. (2008): "Technical versus institutional innovation in Andalusian olive tree orchards: an adoption modelling analysis". $12^{\circ}$ Congreso de la Asociación Europea de Economistas Agrarios, Gante (Bélgica), 26-29 Agosto.

CALATRAVA, J.; FRANCO, J.A. Y GONZÁLEZ, M.C. (2007): "Analysis of the adoption of soil conservation practices in olive groves: the case of mountainous areas in southern Spain". Spanish Journal of Agricultural Research, 5(3): pp 249-258.

CALATRAVA, J.; BARBERÁ, G.G. Y CASTILLO, V.M. (2011): "Farming practices and policy measures for agricultural soil conservation in semi-arid Mediterranean areas: the case of the Guadalentín basin in southeast Spain". Land Degradation and Development, 22: pp 58-69.

CAP (2002): El olivar andaluz, Sevilla: Consejería de Agricultura y Pesca, Junta de Andalucía.

CAP (2006): Anuario de Estadísticas Agrarias y Pesqueras de Andalucía, Sevilla: Consejería de Agricultura y Pesca, Junta de Andalucía.

CARM (2007): Programa de Desarrollo Rural de la Región de Murcia 20072013. Tomo II, Comunidad Autónoma de la Región de Murcia, Murcia.

CARMONA MARTÍNEZ, M.M. Y GÓMEZ GARCÍA, J. (2003): "Difusión de Innovaciones Hospitalarias". Estudios de Economía Aplicada, 21(1): pp 5372.

CARMONA, M.M.; GÓMEZ, J. Y FAURA, U. (2005): "La difusión de la agricultura ecológica en España: una propuesta de modelización matemática". Revista Española de Estudios Agrosociales y Pesqueros, 205: pp 39-63.

CARRILLO M. Y GONZÁLEZ J.M. (2002): "A new approach to modelling sigmoidal curves".Technological Forecasting and Social Change, 69: pp 233-241.

CMA (2002): Usos y coberturas del suelo en Andalucía. 2002, Sevilla: Consejería de Medio Ambiente, Junta de Andalucía.

DIOS PALOMARES, R.; MARTÍNEZ PAZ, J.M. Y VICARIO MODROÑO, V. (2003): "Eficiencia versus innovación en explotaciones agrarias". Estudios de Economía Aplicada, 21(3): pp 485-501.

FEDER, G.; JUST, R.E. Y ZILBERMAN, D. (1985): "Adoption of Agricultural Innovations in Developing Countries: A Survey". Economic Development and Cultural Change, 33: pp 255-298.

FEDER, G. Y UMALI, D.L. (1993): "The adoption of agricultural innovations. A review". Technological Forecasting and Social Change, 43: pp 215-239.

FOURT, L.A. Y WOODLOCK, J.W. (1960): "Early prediction of market success for new grocery products". Journal of Marketing, 25(2): pp 31-38.

FRANCIA-MARTÍNEZ J.R.; DURÁN-ZUAZO V.H. Y MARTÍNEZ-RAYA A. (2006): "Environmental impact from mountainous olive orchards under 
different soil-management systems (SE Spain)". Science of the Total Environment, 358: pp 46-60.

GÓMEZ MUÑOZ, A.C. (1988): "Análisis del comportamiento innovador de los agricultores a través de sus curvas de difusión". Revista de Estudios Agrosociales y Pesqueros, 145: pp 85-106.

GÓMEZ, J.A.; GIRÁLDEZ, J.V.; PASTOR, M. Y FERERES, E. (1999): "Effects of tillage method on soil physical properties, infiltration and yield in an olive orchard". Soil and Tillage Research, 52: 167-175.

GREENE W.H. (2007): Econometric Analysis. Sixth Edition, New Jersey: Prentice Hall.

GRILICHES Z. (1957): "Hybrid corn: an exploration in the economics of technological change". Econometrica, 25: pp 501-522.

GRILICHES Z. (1960): "Hybrid corn and economics of innovation". Science, 132: pp 275-280.

INE (1999): Censo agrario, Madrid: Instituto Nacional de Estadística.

LEATHERS, H.D. Y SMALE, M. (1991): "A Bayesian approach to explaining sequential adoption of components of a technological package". American Journal of Agricultural Economics, 73(3): pp 734-742.

MAHAJAN, V.; BRETSCHNEIDER, S.I. Y BETTIS, R.A. (1988): "The adoption of the m-form organizational structure: a test of imitation hipótesis". Management Science, 34(10): pp 1188-1201.

MAHAJAN, V.; MULLER, E. Y BASS, F.M. (1990): "New products diffusion model in marketing: a review and directions for research". Journal of Marketing, 54: pp 1-26.

MANSFIELD, E. (1961): "Technical change and the rate of imitation". Econométrica, 29: pp 741-766.

MARRA, M.; PANNELL, D.J. Y ABADI, A.K. (2003): "The economics of risk, uncertainty and learning in the adoption of new agricultural technologies: where are we on the learning curve?". Agricultural Systems, 75: pp 215-234.

MILGROOM J.; SORIANO M.A.; GARRIDO J.M.; GÓMEZ J.A. Y FERERES E. (2007): "The influence of a shift from conventional to organic olive farming on soil management and erosion risk in southern Spain". Renewable Agriculture and Food Systems, 22: pp 1-10.

PARRA-LÓPEZ C.; DE HARO-GIMÉNEZ T. Y CALATRAVA-REQUENA J. (2007): "Diffusion and Adoption of Organic Farming in the Southern Spanish Olive Groves". Journal of Sustainable Agriculture, 30(1): pp 105-151.

RATKOWSKY, D.A. (1983): Nonlinear regression modelling, New York: Marcel Dekker.

RODRÍGUEZ-LIZANA, A.; ESPEJO-PÉREZ, A. J.; GONZÁLEZ-FERNÁNDEZ, P. Y ORDÓÑEZ-FERNÁNDEZ, R. (2008): "Pruning Residues as an Alternative to Traditional Tillage to Reduce Erosion and Pollutant Dispersion in Olive Groves" en Water, Air \& Soil Pollution, 193: pp 165-173. 
ROGERS, E. M. (1995): Diffusion of Innovations. $4^{\text {th }}$ Edition, New York: The Free Press.

ROGERS, E.M. (1958): "Categorizing the adopters of agricultural practices". Rural Sociology, 23: pp 346-354.

RYAN B. Y GROSS N.C. (1943): "The diffusion of hybrid seed corn in two lowa communities". Rural Sociology, 8: pp 15-24.

STONEMAN P. (1981): "Intra firm diffusion, bayesian learning and profitability". Economic Journal, 91: pp 375-388. 